\title{
Micronutrientes foliares na goiabeira fertirrigada com biofertilizante e nitrogênio no semiárido
}

\author{
Elisson Alves Santana, Jackson Teixeira Lobo, Renan Nunes Pereira, \\ Augusto Miguel Nascimento Lima, Jaílson Cavalcante Cunha, Ítalo Herbert Lucena Cavalcante*
}

Universidade Federal do Vale do São Francisco, Petrolina, Brasil

*Autor correspondente, e-mail: italo.cavalcante@univasf.edu.br

\section{Resumo}

Demandados em pequenas quantidades, os micronutrientes exercem papel fundamental para o desenvolvimento adequado das plantas e podem ser fornecidos por insumos sintéticos ou orgânicos, como os biofertilizantes. Dessa forma, um experimento foi conduzido entre julho de 2014 e agosto de 2015, na área experimental da Universidade Federal do Vale do São Francisco, Petrolina-PE, para avaliar os teores foliares de micronutrientes em goiabeira Paluma em função de fertirrigação com biofertilizante bovino e adubação nitrogenada. O delineamento experimental foi em blocos casualizados em esquema fatorial $(5 \times 2)$ referentes às concentrações de biofertilizante bovino $(0,2,5,5,0,7,5$ e 10\%) e adubação mineral com nitrogênio $(50 \%$ e $100 \%$ do recomendado segundo a demanda da cultura), com quatro repetições e cinco plantas por parcela. Apenas os teores foliares de Mn são afetados pelo biofertilizante bovino em goiabeiras fertirrigadas com 100\% de N. Os teores foliares de todos os micronutrientes (Mn, B, Fe e Zn) não são influenciados pela fertirrigação nitrogenada. O biofertilizante é eficiente no fornecimento de Fe.

Palavras-chave: Psidium guajava L., matéria orgânica, nutrição de plantas

\section{Leaf micronutrients in guava fertigated with biofertilizer and nitrogen in semiarid}

\begin{abstract}
Required in small amounts, micronutrients play a key role for adequate plant development, which can be supplied by synthetic or organic fertilizers, such as biofertilizers. Thus, an experiment was conducted between July 2014 and August 2015, in the experimental area of the Federal University of the São Francisco Valley, Petrolina-PE, Brazil, to evaluate the micronutrient leaf concentrations of Paluma guava as a function of fertigation with bovine biofertilizer and Nitrogen fertilization. The experimental design was a randomized complete block design $(5 \times 2)$ referring to the concentrations of bovine biofertilizer $(0,2.5,5.0,7.5$ and $10 \%)$ and $\mathrm{N}$-fertilizing $(50 \%$ and $100 \%$ of $\mathrm{N}$ dose following plant demand), with four replicates and five plants per plot. Only the leaf Mn concentrations are affected by bovine biofertilizer in guava trees fertigated with $100 \%$ of recommended N. Leaf concentrations of all the micronutrients ( $\mathrm{Mn}, \mathrm{B}, \mathrm{Fe}$ and $\mathrm{Zn}$ ) are not influenced by nitrogen fertigation. The biofertilizer is efficient in providing Fe.
\end{abstract}

Keywords: Psidium guajava L., organic matter, plant nutrition 
A goiabeira (Psidium guajava L.) é uma planta originária do Norte da América do Sul, pertence à família Myrtaceae, presente principalmente em regiões tropicais e subtropicais (Cavalcante et al., 2010).No Brasil, aproximadamente $32 \%$ da produção de goiaba é proveniente de regiões de clima semiárido (IBGE, 2016).

Na região Nordeste, a região semiárida beneficiada pela bacia do rio São Francisco concentra importantes áreas produtivas de goiaba, pois possui além de disponibilidade hídrica, as condições de clima e solo favoráveis ao cultivo (Rodrigues et al., 2015).

No cultivo da goiabeira, o manejo adequado da fertilidade do solo é fundamental para que a absorção dos nutrientes seja realizada de forma equilibrada refletindo no incremento da produtividade da cultura (Cavalcante et al., 2008a). Os solos tropicais são em sua maioria intensamente intemperizados, demandando fornecimento de matéria orgânica para melhoria da capacidade produtiva em decorrência dos efeitos positivos na disponibilidade de nutrientes, estrutura, infiltração e retenção de água, atividade e biomassa microbiana do solo (Cunha et al., 2012, Alencar et al., 2016).

Em substituição ou associados às tradicionais adubações sintéticas, os biofertilizantes são alternativa viável e de rápida disponibilização de nutrientes, sendo utilizados na produção de diversas plantas frutíferas (Sharma et al., 2011, Dantas et al., 2015), devido ser rico em matéria orgânica, proveniente de processo fermentativo e encontrar-se em estado líquido, - que torna sua aplicação através do sistema de fertirrigação simplificada e menos onerosa (Gross et al., 2008, Ramniwas et al., 2013).

Assim como os macronutrientes, os micronutrientes são fundamentais ao desenvolvimento vegetal, e quando fornecidos em teores inadequados retardam o crescimento das plantas, podendo ocasionar desequilíbrios no metabolismo vegetal, tornando as plantas mais sensíveis ao ataque de pragas e doenças reduzindo a eficiência de uso dos fertilizantes contendo macronutrientes (Lima Neto et al., 2015).

Apesar da grande importância da cultura da goiabeira, são poucas as informações sobre o sev estado nutricional em micronutrientes associado ao manejo da fertilidade do solo para o aumento da produção de frutos com qualidade (Rocha et al., 2016).

Neste sentido, o presente estudo teve como objetivo avaliar os teores foliares de micronutrientes da goiabeira em função da fertirrigação com biofertilizante e nitrogênio no semiárido brasileiro.

O experimento foi conduzido entre julho de 2014 e agosto de 2015 (dois ciclos consecutivos) na área experimental da Universidade Federal do Vale do São Francisco, em Petrolina-PE. O clima da região é semiárido, classificado como Bswh (Köeppen), o solo é um Argissolo Amarelo (Ultisol - Classificação Americana de taxonomia do solo), os resultados da análise dos atributos físicos e químicos constam na Tabela 1.

Tabela 1. Características químicas e físicas do solo (0-20 e 20-40 cm de profundidade), da área experimental antes da instalação do experimento.

\begin{tabular}{|c|c|c|}
\hline \multirow{2}{*}{ Característica do solo } & \multicolumn{2}{|c|}{ Valor } \\
\hline & $0-20 \mathrm{~cm}$ & $20-40 \mathrm{~cm}$ \\
\hline $\mathrm{pH}$ (em água) & 6,2 & 5,4 \\
\hline $\mathrm{Ca}^{2+}\left(\mathrm{cmol}_{\mathrm{c}} \mathrm{dm}^{3}\right)$ & 2,1 & 2,0 \\
\hline $\mathrm{Mg}^{2+}\left(\mathrm{cmol}_{\mathrm{c}} \mathrm{dm}^{3}\right)$ & 1,4 & 1,2 \\
\hline $\mathrm{Al}^{3+}\left(\mathrm{cmol}_{\mathrm{c}} \mathrm{dm}^{3}\right)$ & 0,0 & 0,0 \\
\hline $\mathrm{K}^{+}\left(\mathrm{cmol}_{\mathrm{c}} \mathrm{dm}^{3}\right)$ & 0,74 & 0,63 \\
\hline $\mathrm{Na}^{+}\left(\mathrm{cmol} \mathrm{dm}^{3}\right)$ & 0,11 & 0,19 \\
\hline $\mathrm{P}\left(\mathrm{mg} / \mathrm{dm}^{3}\right)$ & 207,0 & 58,0 \\
\hline Matéria orgânica (\%) & 0,56 & 0,53 \\
\hline Argila $\left(\mathrm{g} \mathrm{kg}^{-1}\right)$ & 95 & 11 \\
\hline Silte $\left(\mathrm{g} \mathrm{kg}^{-1}\right)$ & 32 & 60 \\
\hline Areia $\left(\mathrm{g} \mathrm{kg}^{-1}\right)$ & 870 & 774 \\
\hline
\end{tabular}

Pe K: Melich-1; Ca e Mg: KCl $1 \mathrm{M}$ 
O pomar foi formado por goiabeiras da cultivar Paluma propagadas por estaquia, com um ano de idade, cultivadas no espaçamento $4 \times 4 \mathrm{~m}$, irrigadas diariamente com um emissor por planta com vazão de $42 \mathrm{~L} \mathrm{~h}^{-1}$ com base nos registro de evapotranspiração diária e corrigidas de acordo com o coeficiente de cultura $(\mathrm{Kc})$. Todas as práticas de manejo para a poda controle de plantas daninhas, pragas e doenças foram realizadas seguindo as instruções de Natale et al. (2009).

O delineamento experimental adotado foi em blocos casualizados com tratamentos distribuídos em esquema fatorial $(5 \times 2)$ referentes às concentrações de biofertilizante $[0,2,5,5,0$, 7,5 e 10\%] e adubação nitrogenada $\quad(50 \%$ e $100 \%$ do $\mathrm{N}$ recomendado segundo a demanda da cultura), com quatro repetições de cinco plantas cada.

O biofertilizante utilizado no experimento foi constituído de esterco e água, na razão 1:1 (em volume), submetido à fermentação anaeróbica por 30 dias. A aplicação do biofertilizante foi feita quinzenalmente em uma área de solo de $0,283 \mathrm{~m}^{2}$, em torno da base da planta por meio da água da irrigação, cada planta recebeu 2,4 $\mathrm{L} \mathrm{m}^{-2}$ por copa, de acordo com recomendação de Cavalcante et al. (2008a).O biofertilizante continha 0,72 $\mathrm{g} \mathrm{L}^{-1}$ de $\mathrm{N}, 0,04 \mathrm{~g} \mathrm{~L}^{-1}$ de P, 0,50 $\mathrm{g} \mathrm{L}^{-1}$ de $\mathrm{K}, 0,20 \mathrm{~g} \mathrm{~L}^{-1}$ de $\mathrm{Ca}, 0,12 \mathrm{~g} \mathrm{~L}^{-1}$ de $\mathrm{Mg}, 0,39 \mathrm{~g} \mathrm{~L}^{-1}$ de $S, 4,0 \mathrm{mg} \mathrm{L}^{-1}$ de $B, 6,0 \mathrm{mg} \mathrm{L}^{-1}$ de $\mathrm{Cu}, 77 \mathrm{mg} \mathrm{L}^{-1}$ de Fe, $10 \mathrm{mg} \mathrm{L}^{-1} \mathrm{de} \mathrm{Mn}, 16 \mathrm{mg} \mathrm{L}^{-1}$ de $\mathrm{Zn}$ e $81 \mathrm{mg}$ $\mathrm{L}^{-1}$ de sódio.

As demais adubações foram realizadas quinzenalmente através do sistema de fertirrigação, baseadas na análise de solo e demanda da cultura, iniciando após a poda de produção e continuando até 20 dias antes da colheita, usando fertilizante formulado composto por $12 \%$ de $N, 5 \%$ de $P, 11 \%$ de $K, 13,1 \%$ de Ca e $0,2 \%$ de $B$.

Para as análises dos teores de micronutrientes foliares, quatro repetições de 60 folhas (em cada repetição, 12 folhas por planta sendo 5 plantas amostradas) foram coletadas a partir de ramos terminais sem frutos, da parte média do dossel, na floração. De acordo com as recomendações de Natalie\& Prado (2002), o terceiro par de folhas a partir do ápice dos brotos foi colhido para realizar a análise nutricional.

$$
\text { As folhas foram analisadas }
$$
quimicamente após a lavagem com água destilada e secagem a $70{ }^{\circ} \mathrm{C}$ durante $48 \mathrm{~h}$. Os teores de $\mathrm{B}$ foram determinados pelo método de espectrofotometria de absorção molecular e os teores de $\mathrm{Zn}, \mathrm{Mn}$ e Fe foram determinados por espectrofotometria de absorção atômica (Chapman \& Pratt, 1961).

Os dados foram submetidos à análise de variância (ANOVA) utilizando-se o teste ' $F$ ' da ANOVA, as médias do fertilizante $N$ foram comparadas pelo teste de Tukey e as doses de biofertilizante foram submetidas à análise de regressão com dados combinados dos ensaios consecutivos. As análises estatísticas foram realizadas utilizando os softwares Sisvar 5.3 e SigmaPlot 10.0, e os resultados considerados significativos a $5 \%$ de probabilidade.

Os teores foliares dos micronutrientes avaliados não foram influenciados significativamente pelas doses de biofertilizante e de nitrogênio estudadas, assim como a interação entre os fatores não foi significativa, com exceção para o manganês (Tabela 2).

Os teores foliares de $\mathrm{Mn}$ sob adubação com $50 \%$ da recomendação de adubação nitrogenada não apresentaram ajuste significativo a nenhum modelo de regressão, enquanto que o $\mathrm{Mn}$ associado à dose de $100 \%$ houve ajuste ao modelo quadrático (Figura 1).

De acordo com Natale et al. (2002), a extração de micronutrientes pela cultivar Paluma, ocorre em ordem crescente: B, Cu, Zn, Fe e Mn. Os mesmos autores determinaram o conteúdo $\left(\mathrm{mg} \mathrm{kg}^{-1}\right)$ adequado de micronutrientes foliar em goiabeiras após terceiro ciclo, B (20-25), Cu (2040), Zn (25-35), Fe (60-90) e Mn (40-80).

As se considerar a faixa de suficiência definida por Natale et al. (2002), todos os tratamentos encontraram-se acima da faixa suficiente para $\mathrm{B}$ e $\mathrm{Fe}$, porém deficientes em $\mathrm{Zn}$ e $\mathrm{Mn}$, resultados que concordam com os de Cavalcante et al. (2008a), que verificaram para o maracujá um efeito positivo do uso de biofertilizante simples apenas nos teores de B, $\mathrm{Fe}$ e $\mathrm{Cu}$, indicando dessa forma a necessidade de suplementação ao biofertilizante para que se obtenha fornecimentos adequados desses 
Tabela 2. Análise de variância para teores foliares (Mn, Zn, Fe e B) da goiabeira Paluma em função da adubação nitrogenada e doses de biofertilizante bovino fornecidos via fertirrigação.

\begin{tabular}{|c|c|c|c|c|}
\hline Fonte de variação & $M n\left(\mathrm{mg} \mathrm{kg}^{-1}\right)$ & $\mathrm{Zn}\left(\mathrm{mgkg}^{-1}\right)$ & $\mathrm{Fe}\left(\mathrm{mgkg}^{-1}\right)$ & $\mathrm{B}\left(\mathrm{mgkg}^{-1}\right)$ \\
\hline Adub. Nitrogenada (N) & $0,12^{\text {ns }}$ & $0,06^{\text {ns }}$ & $1,09^{\text {ns }}$ & $0,03^{\text {ns }}$ \\
\hline $50 \%$ & 11,40 & 3,94 & 77,60 & 28,73 \\
\hline $100 \%$ & 10,57 & 4,03 & 93,00 & 28,23 \\
\hline $\mathrm{DMS}$ & 4,89 & 0,73 & 30,39 & 5,98 \\
\hline Doses Biofertilizante (B) & $1,77^{\text {ns }}$ & $2,02^{\text {ns }}$ & $1,30^{\text {ns }}$ & $0,29^{\text {ns }}$ \\
\hline $0 \%$ (testemunha) & 12,05 & 3,95 & 71,72 & 30,79 \\
\hline $2,5 \%$ & 14,32 & 4,20 & 62,94 & 30,55 \\
\hline $5,0 \%$ & 9,50 & 4,50 & 109,08 & 27,15 \\
\hline $7,5 \%$ & 13,36 & 4,17 & 95,14 & 27,15 \\
\hline $10,0 \%$ & 6,03 & 3,10 & 84,68 & 27,80 \\
\hline Interação N X B & $4,19^{*}$ & $1,53^{\text {ns }}$ & $1,24^{\text {ns }}$ & $0,26^{\text {ns }}$ \\
\hline C.V. (\%) & 64,53 & 26,45 & 50,95 & 30,35 \\
\hline
\end{tabular}

DMS = diferença mínima significativa; C.V. = coeficiente de variação; " ns: não significativo;" significativo ao nivel de 5\% de probabilidade.

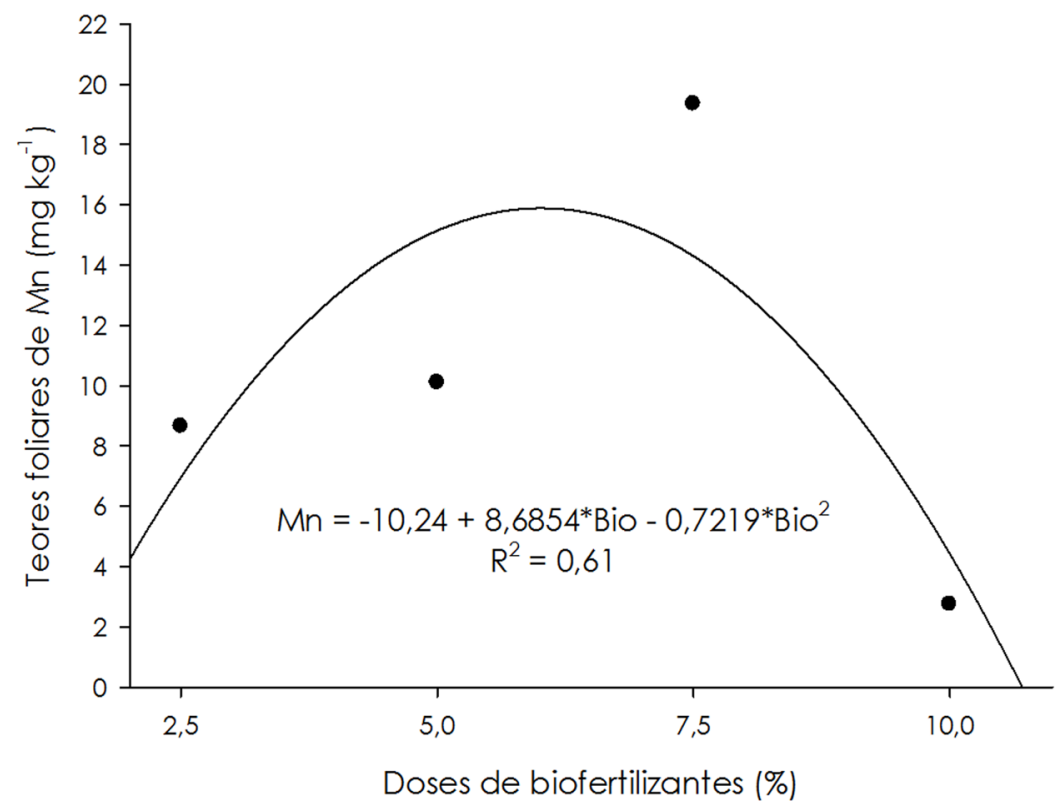

Figura 1. Teores foliares de manganês na goiabeira em função de biofertilizante e fertilizante N (100\% da recomendação de N da cultura).

micronutrientes. Apesar dos elevados teores de $B$ e Fe registrados no trabalho, não se verificaram sintomas visuais de toxidez.

Apesar da pequena concentração de Mn do biofertilizante bovino fornecido às plantas (10 mg L-1), o máximo acúmulo do micronutriente nas plantas não ocorreu para a maior dose do insumo aplicada, indicando que além da quantidade reduzida, houve dificuldade na sua absorção pela planta.

Em relação ao efeito não significativo das doses de nitrogênio estudadas, Cavalcante et al. (2008b), estudando a composição mineral de folhas de goiabeira paluma em função da aplicação via irrigação de doses semanais de 0 até $5 \mathrm{~kg} \mathrm{ha}^{-1}$ de sulfato de amônio, também não verificaram efeito significativo para os teores foliares de boro e zinco. Em contrapartida, Amorim et al. (2015) estudando diferentes doses de nitrogênio e potássio em goiabeira constataram que a adubação nitrogenada promoveu aumento nos teores foliares de $\mathrm{Mn}$ e decréscimos de $B$.

Destaca-se que o manejo de micronutrientes não é simples devido às pequenas quantidades exigidas pela cultura, à estreita faixa entre suficiência e toxidez e à influência de fatores de solo quanto à disponibilidade às plantas. 


\section{Referências}

Alencar, R.D., Leite, G.A., Mendonça, V.M, Lima, F.V., Pereira, G.A., Farias, W.C. 2016. Potassium fertilization influencing the production and postharvest quality of 'Paluma' guava grown under semiarid conditions at Rio Grande do Norte, Brazil. Comunicata Scientiae 7: 139-148.

Amorim, D.A., Souza, H.A., Rozane, D.E., Montes, R. M., Natale, W. 2015. Adubação nitrogenada e potássica em goiabeiras 'Paluma': efeito no estado nutricional das plantas. Revista Brasileira de Fruticultura 37: 210-219.

Cavalcante, Í.H.L., Silva, G.F., Cavalcante, L.F., Santos, D., Beckmann-Cavalcante, M.Z. 2008b. Composição mineral de folhas de goiabeira Paluma em função da adubação sulfatonitrogenada. Revista Brasileira de Ciências Agrárias 3: 6-12.

Cavalcante, L.F., Cavalcante, Í.H.L., Santos, G.D. 2008a. Micronutrient and sodium foliar contents of yellow passion plants as function of biofertilizers. Fruits 60: 1-8.

Cavalcante, L.F., Vieira, M.S., Santos, A.F., Oliveira, W.M., Nascimento, E.A.M. 2010. Água salina e esterco bovino líquido na formação de mudas de goiabeira cultivar Paluma. Revista Brasileira de Fruticultura 32: 251-261.

Chapman, H.D., Pratt, P.F. 1961. Method of analysis for soils, plants and waters. Agriculture \& Natural Resources, Berkeley, USA, 68p.

Cunha, E.Q., Stone, L.F., Ferreira, E.P.B., Didonet, E.D., Moreira, J.A.A. 2012. Atributos físicos, químicos e biológicos de solo sob produção orgânica impactados por sistema de cultivo. Revista Brasileira de Engenharia Agrícola e Ambiental 16: 56-63.

Dantas S.A.G., Cavalcante, L.F., Alves, E.U., Nascimento, J.A.M., Silva, S.A., Dantas, T.A.G. 2015. Physiological quality of yellow passion fruit seed produced under saline water, NPK and bovine biofertilizer. African Journal of Agricultural Research 10: 2948-2954.

Gross, A., Arusi, R., Fine, P., Nejidat, A. 2008. Assessment of extraction methods witf fowl manure for the production of liquid organic fertilizers. Bioresource Technology 99: 327-334.

IBGE. Banco de dados agregados do Instituto Brasileiro de Geografia e Estatística. 2014. http:// www.sidra.ibge.gov.br <Acesso em: 13 Abr. 2016>

Lima Neto, A.J., Natale, W., Modesto, V.C. 2015. Resposta de porta-enxertos de caramboleira à adubação com zinco. Revista Brasileira de Fruticultura 37: 1074-1082.
Natale, W., Coutinho, E.L.M., Pereira, F.M., Boaretto, A.E. 2002. Nutrients foliar content for high productivity cultivars of guava in Brazil. Acta Horticulturae 594: 383-386.

Natale, W., Prado, R.M. 2002. Fertirrigação em goiabeira. Unesp, Jaboticabal, Brasil. 41 p.

Natale, W., Rozane, D.E., Souza, H.A., Amorim, D.A. 2009. Cultura da goiaba: do plantio à comercialização. Unesp, Jaboticabal, Brasil. 573 p.

Ramniwas, K.R.A., Pareek, S.D.K., Singh, V. 2013. Effect of drip fertigation scheduling on fertilizer use efficiency, leaf nutrient status, yield and quality of 'shweta' guava (Psidium guajava L.) under meadow orcharding. National Academy Science Letters 36: 483-488.

Rocha, L.F., Cavalcante, L.F., Nunes, J.C., Souto, A.G.L., Cavalcante, A.C.P., Cavalcante, Í.H.L., Pereira, W.E. 2016. Fruit production and quality of guava Paluma as a function of humic substances and soil mulching. African Journal of Biotechnology 15: 1962-1969.

Rodrigues, M.S., Santana, M.C., Uchoa, A.L.P., Menezes, A.X.S.M., Cavalcante, Í.H.L., Lima, A.M.N. 2015. Delineation of management zones based on soil physical attributes in an irrigated guava field in the Semi-Arid region, Brazil. African Journal of Agricultural Research 10: 4185-4192.

Sharma, A., Wali, V.K., Bakshi, P., Jamwal, M. 2011. Effect of organic manures and biofertilizers on leaf and fruit nutrient status in guava (Psidium guajava L.) CV. Sardar. Journal of Horticultural Science 6: 169-171. 